\title{
ANALYSIS OF THERMAL PROPERTIES OF MILITARY JET FUEL AND MODEL MIXTURES SYSTEMS
}

Dianne J. Luning Prak, Paul C. Trulove, David P. Durkin Chemistry Dept

United States Naval Academy

March 2020 


\section{Acknowledgments}

- Midn Ladavish Dorn

- Prof. Jim Cowart

Mechanical Engineering Dept

- Dr. Matt Foley

Naval Academy Preparatory School

- Funding:

Office of Naval Research,

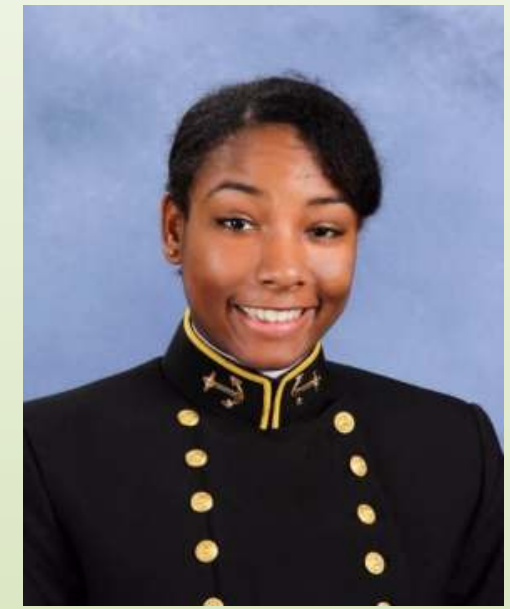

Maria Medieros

Work recently accepted for publication in

Energy and Fuels,

DOI:10.1021/acsenergyfuels.9b04028

Ladavish

Dorn

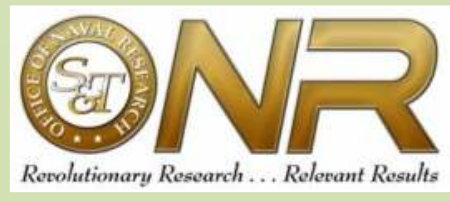




\section{Introduction: Fuel Injection}

Fuel Compression by pressure pulse Depends on bulk modulus $=\rho c^{2}$

Evaporation

Heat capacity, heat of

vaporization
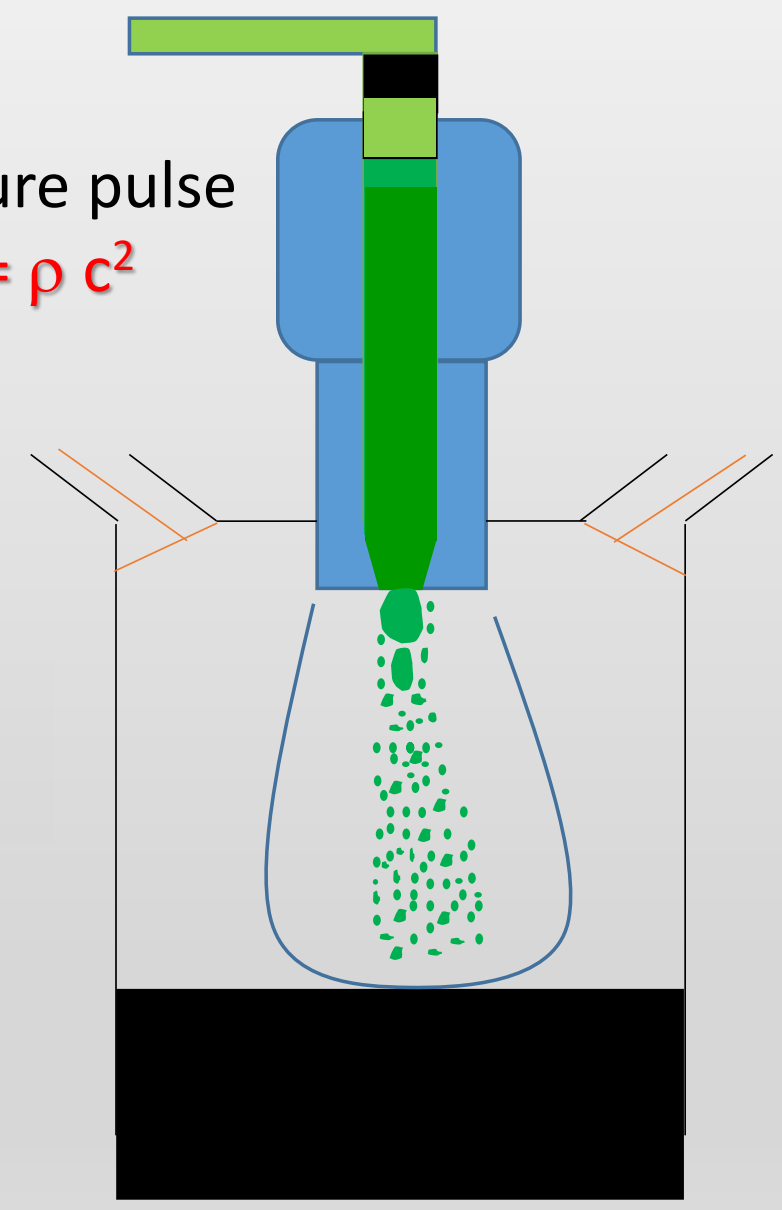

Spray behavior depends on Density, viscosity, surface tension

Reaction in vapor phase 


\section{Introduction: Fuel Injection}

Fuel Compression

Spray behavior

Evaporation Heat capacity, heat of vaporization

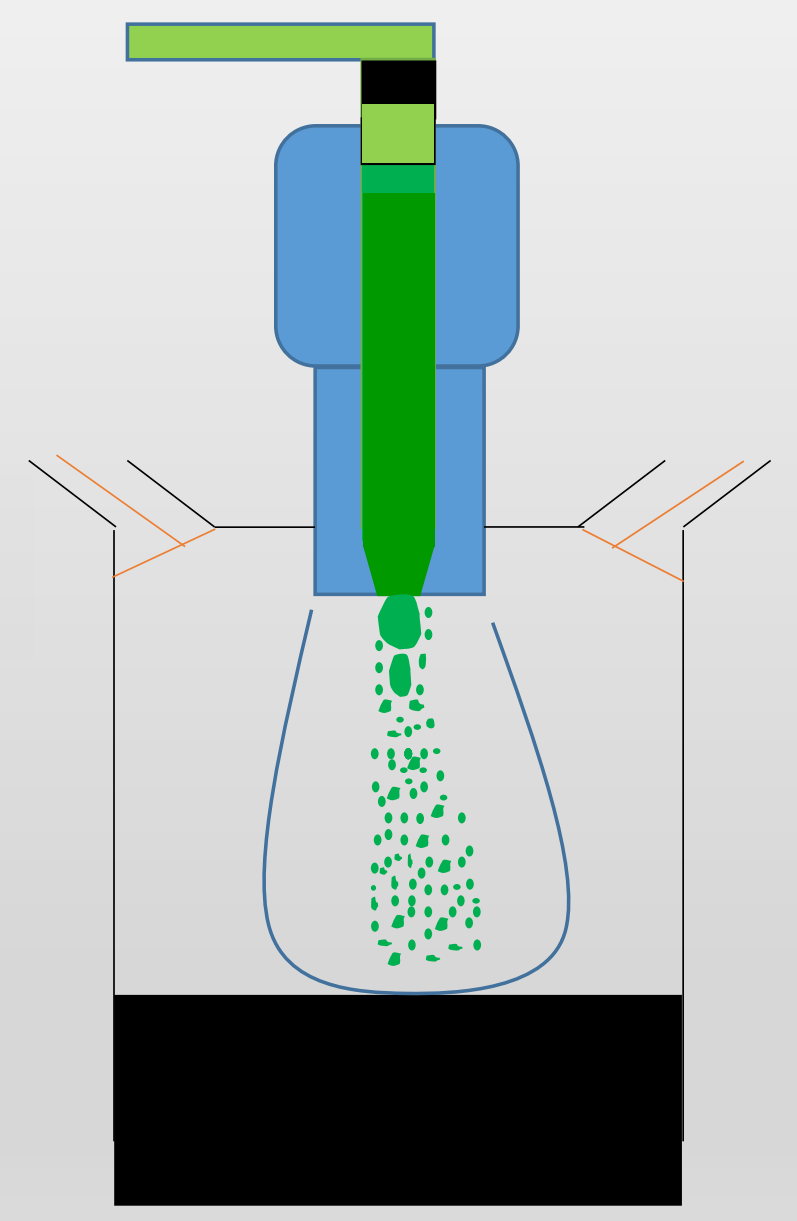

Reaction in vapor phase

Chemical ignition delay

delay 


\section{Introduction - Goals}

- Develop a method to determine the heat of vaporization of military jet fuel, JP-5

- Isothermal methods $\rightarrow$ used in gasoline work/ethanol blends (Fioroni et al. Energy Fuels 2018)

- Temperature ramping methods

- Measure heat of vaporization of jet fuel and surrogate mixtures (mixtures containing a few compounds whose properties match those of the fuel of interest)

- Compounds found in the original fuel

- 6 Surrogates for JP-5 - binary mixtures

- 9 Surrogates for JP-5 - previously published

Luning Prak et al., J. Chem. Eng. Data 2019, 64, 1725-1745.

Cowart, J. et al. , Fuel 2019, 249, 80-88. 


\section{Results GC x GC FID Chemical Composition of JP-5}

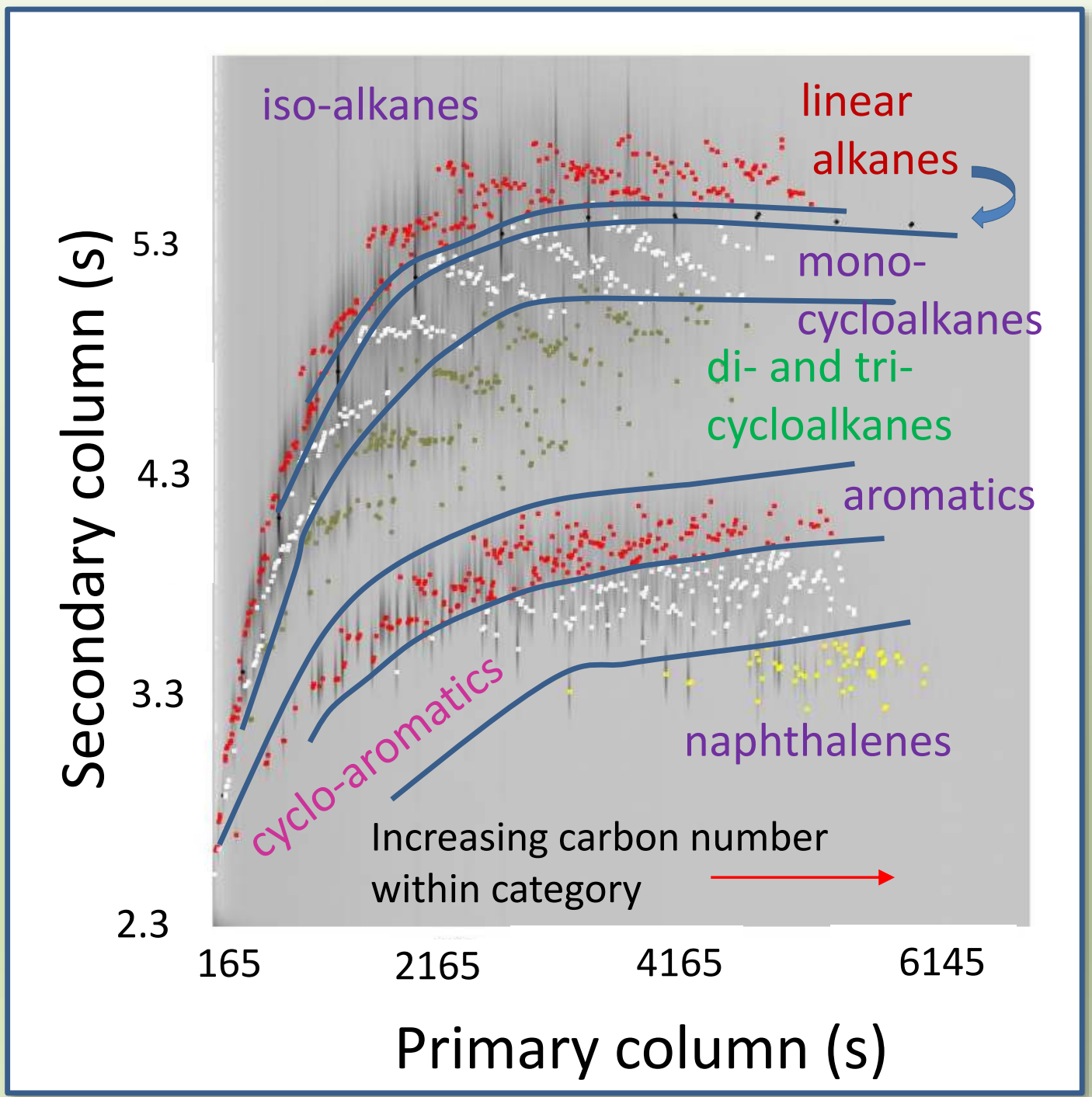

Hundreds of compounds

Categories Iso-alkanes: $\quad 32 \%$ Linear alkanes: $21 \%$ Cycloalkanes: $29 \%$ Aromatics: $\quad 18 \%$

Also used GC/MS Luning Prak et al., J. Chem. Eng. Data 2019, 64, 1725-1745. 


\section{Surrogate mixtures (mass fractions) tested}

$\begin{array}{lllll}\text { Mix } & \mathrm{BB} & \mathrm{BC} & \text { Isocet } & \mathrm{DD} \\ \mathrm{M} 1 & 0.500 & 0.500 & & \\ \mathrm{M} 2 & 0.500 & & 0.500 & \\ \mathrm{M} 3 & 0.500 & & & 0.500 \\ \mathrm{M} 4 & & 0.500 & 0.500 & \\ \mathrm{M} 5 & & 0.500 & & 0.500 \\ \mathrm{M} 6 & & & 0.500 & 0.500 \\ \mathrm{M} 7 & 0.165 & 0.346 & 0.279 & 0.210 \\ \mathrm{M} 8 & 0.245 & 0.170 & 0.275 & 0.310 \\ \mathrm{M} 9 & 0.200 & 0.210 & 0.337 & 0.253 \\ \mathrm{M} 10 & 0.218 & 0.228 & 0.276 & 0.277 \\ \mathrm{M} 11 & 0.079 & 0.365 & 0.459 & 0.098 \\ \mathrm{M} 12 & 0.152 & 0.239 & 0.513 & 0.096 \\ \mathrm{M} 13 & 0.124 & 0.341 & 0.276 & 0.259 \\ \mathrm{M} 14 & 0.125 & 0.348 & 0.211 & 0.316 \\ \mathrm{M} 15 & 0.078 & 0.372 & 0.400 & 0.151 \\ & & & & \end{array}$




\section{Method Development}

Differential Scanning Calorimeter/Thermal Gravimetric Analysis

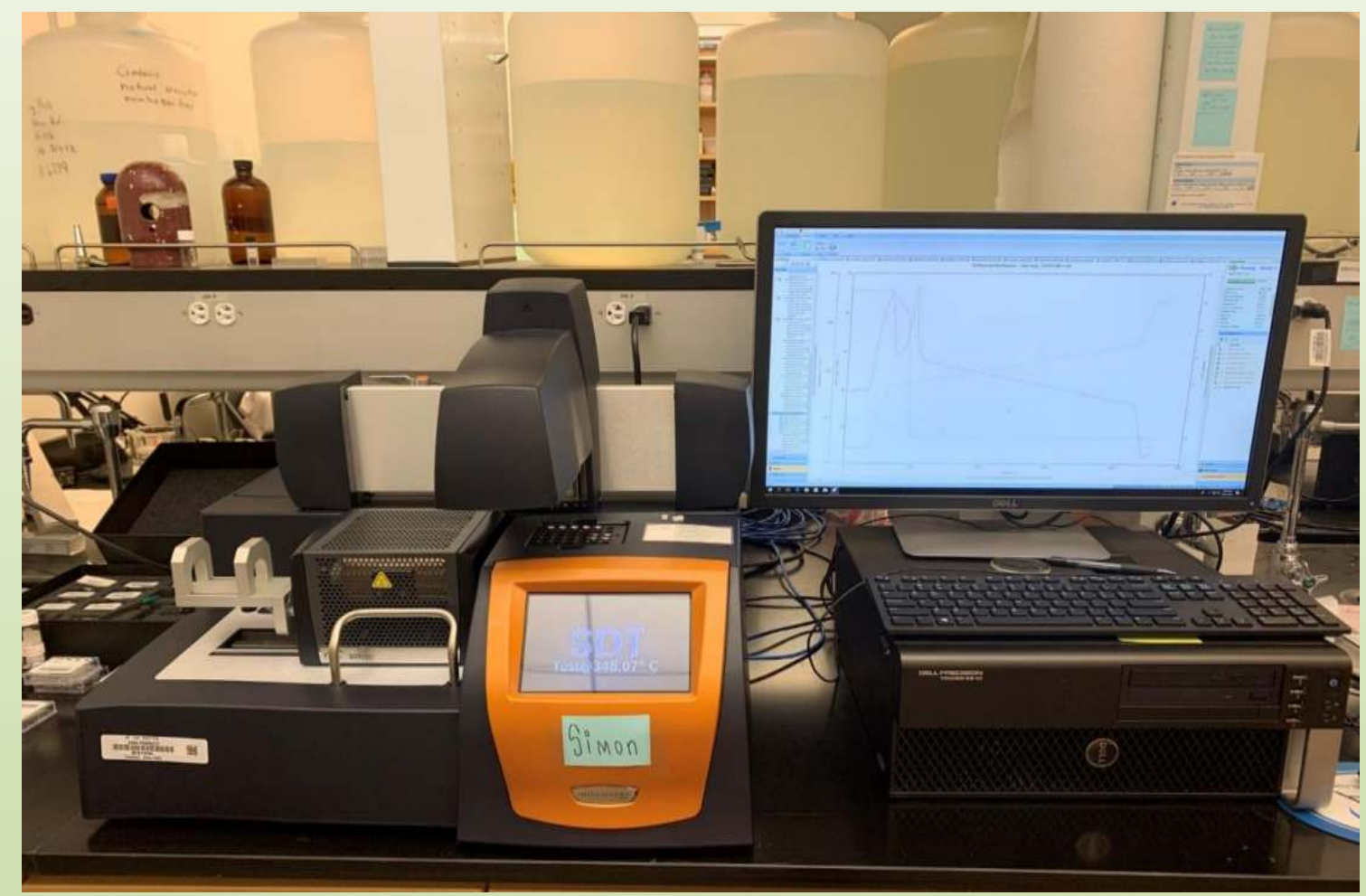

Al pans

hermetically sealed

- Al lid with 75-mm diameter pin-hole

$>$ Isothermal

$>$ Temperature ramping methods

Differential Scanning Calorimeter - used to determine heat capacities (ASTM E1260-11): Al lid with and without pin hole 


\section{Isothermal methods: Individual compounds}

Dodecane heated to $205^{\circ} \mathrm{C}$

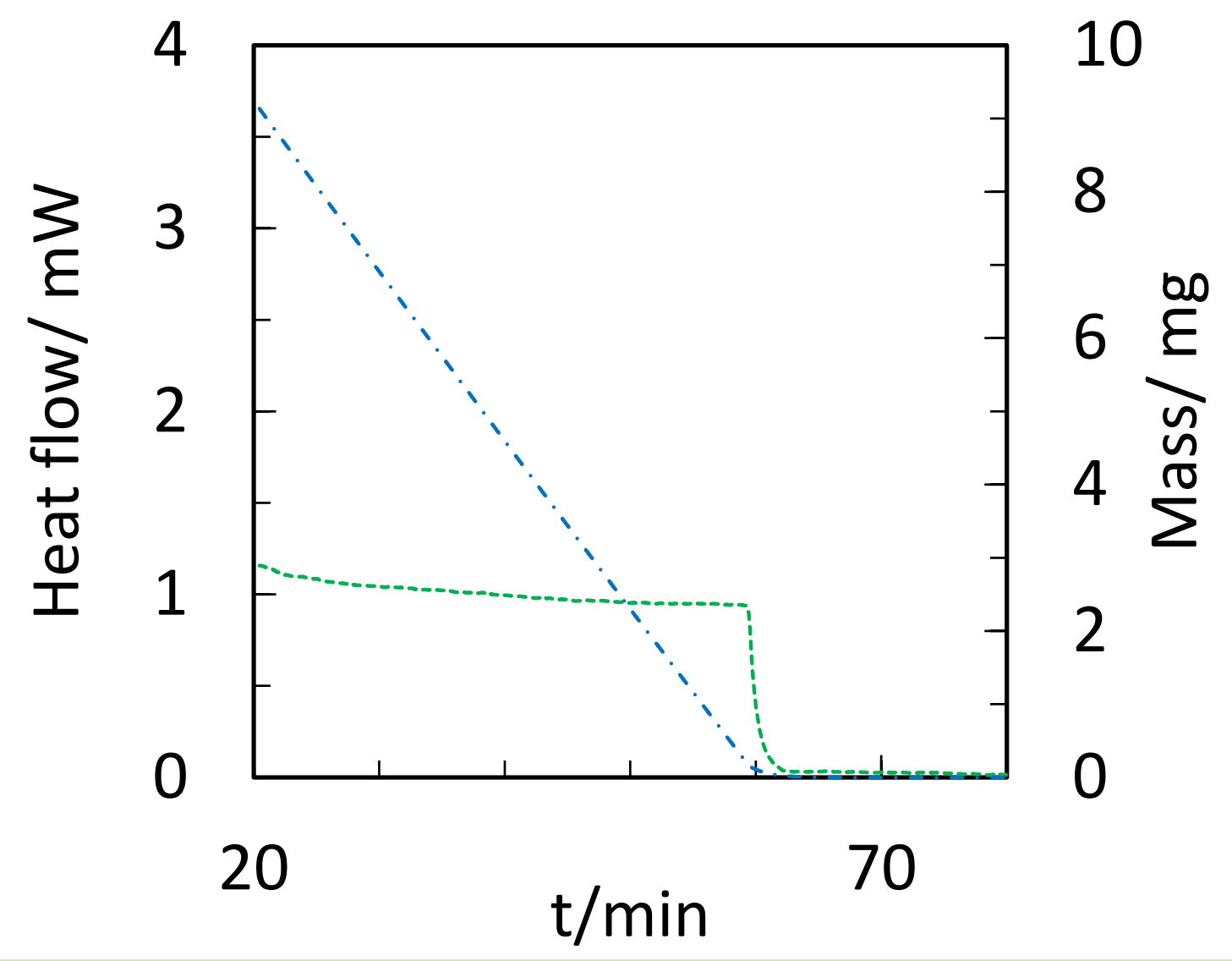

$20{ }^{\circ} \mathrm{C}$ did not work.

Heated to $5-10{ }^{\circ} \mathrm{C}$ below boiling point

Cell correction factor $=0.95$ based on butylbenzene heat of vaporization

HOV of

- Dodecane

- Isocetane

- butylcyclohexane consistent with literature values 


\section{Temperature ramping methods}

- Isothermal methods $\rightarrow$ don't work for jet fuel

- Temperature ramping method

- High-resolution dynamic method

(system changes temp.)

- Method for ramping

equilibrate at $35^{\circ} \mathrm{C}$ for $5 \mathrm{~min}$ heat at $30^{\circ} \mathrm{C} \mathrm{min}^{-1}$ to $170^{\circ} \mathrm{C}$ heat at $1.5^{\circ} \mathrm{C} \mathrm{min}^{-1}$ to $260^{\circ} \mathrm{C}$ heat at $30^{\circ} \mathrm{C} \mathrm{min}^{-1}$ to $350^{\circ} \mathrm{C}$ hold isothermal for $5 \mathrm{~min}$
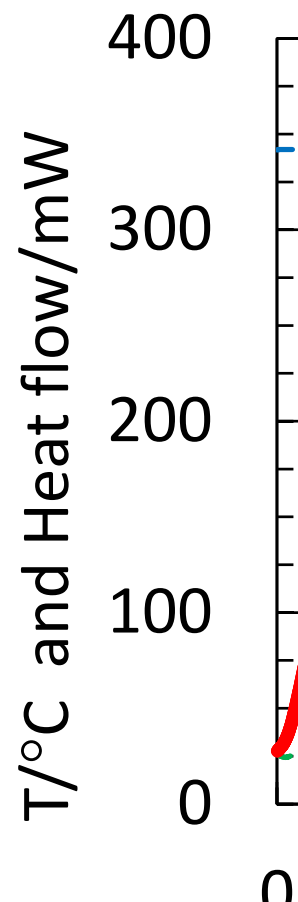

0

$t / \min$ 


\section{Temperature ramping: individual compounds}

Dodecane heated using ramping protocol

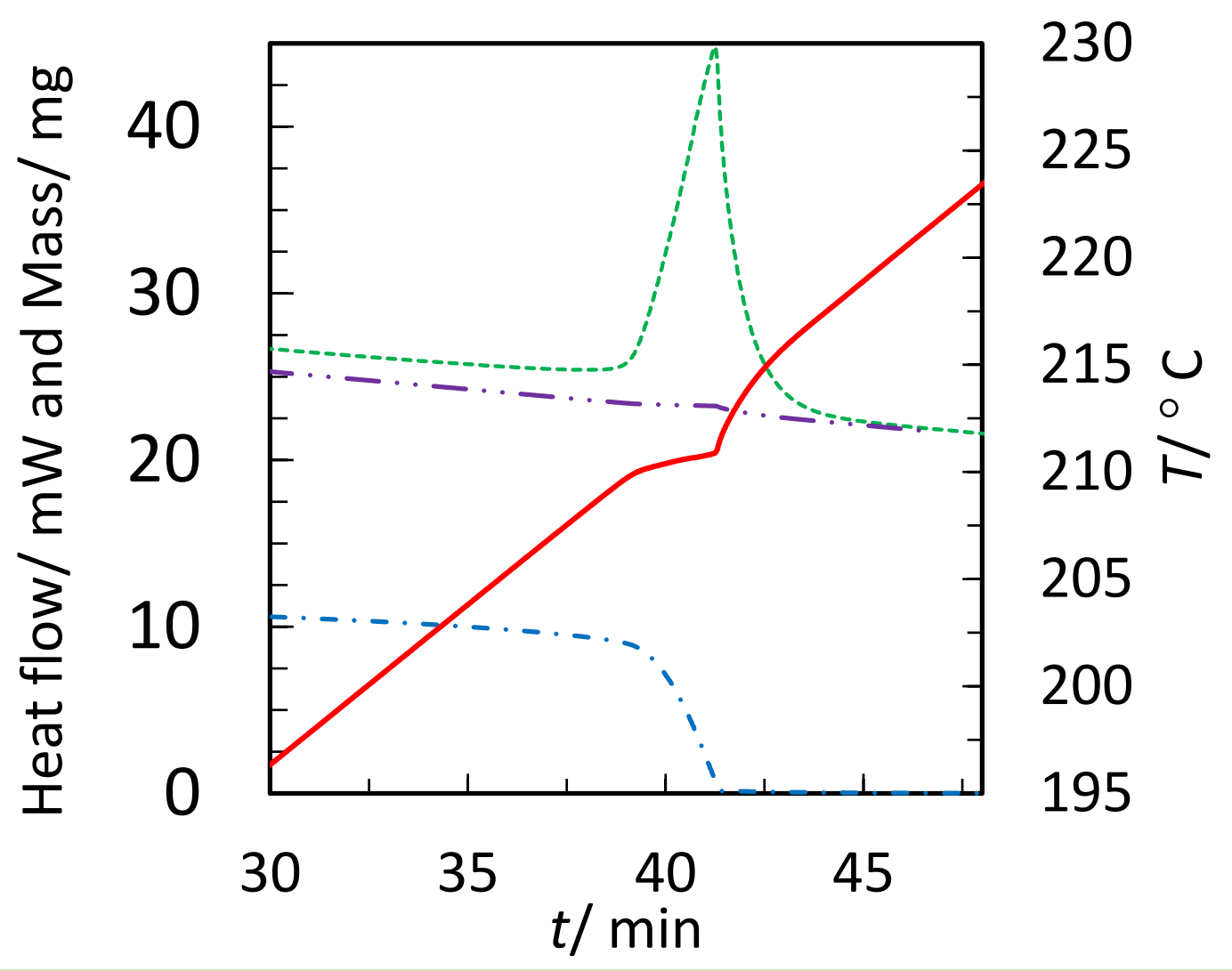

Heat for DSC/TGA Heat to raise temp (from heat capacity)

HOV of

- Dodecane

- Isocetane

- butylbenzene

- butylcyclohexane consistent with literature values

Some lower, some higher than isothermal 


\section{Temperature ramping: JP-5}

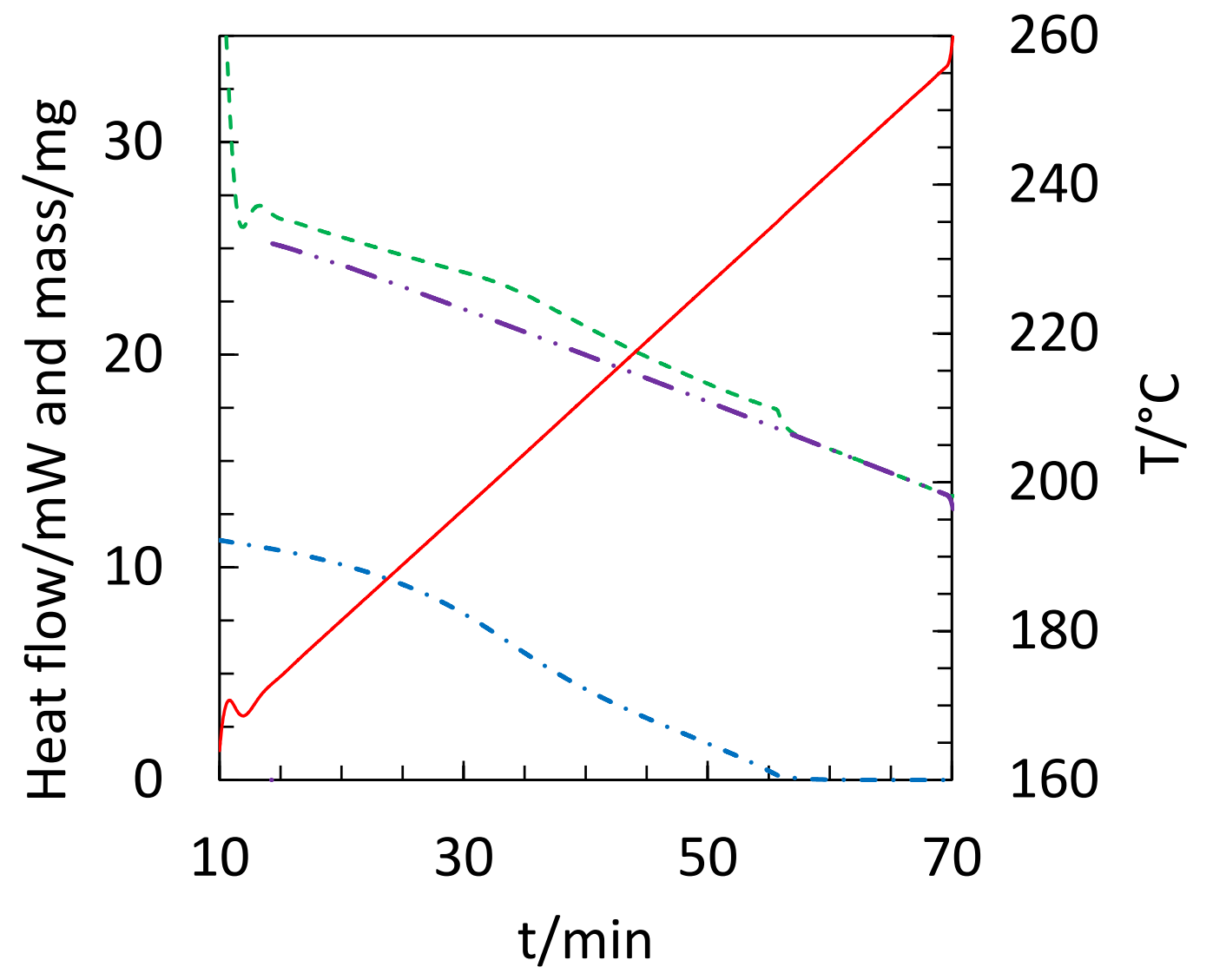

Heat for DSC/TGA heat to raise temp (from heat capacity)

HOV of JP-5:

$239 \pm 8 \mathrm{~J} \cdot \mathrm{g}^{-1}$

\section{Correlations:}

Lefebvre \& Ballal: $214 \mathrm{~J} \cdot \mathrm{g}^{-1}$

Riazi: 231 to $238 \mathrm{~J} \cdot \mathrm{g}^{-1}$

Nelson: $267 \mathrm{~J} \cdot \mathrm{g}^{-1}$

Lefebvre, A. H.; Ballal, D. R., Gas Turbine Combustion: Alternative Fuels and Emissions. 3rd ed.; CRC Press: Boca Raton, FI, 2010. Riazi, M. R., Characterization and Properties of Petroleum Fractions. ASTM International: West Conshohocken, PA, 2005; p 421 Nelson, W. L., Petroleum Refinery Engineering. McGraw-Hill Book Co: New York, 1958. 


\section{Temperature ramping: surrogate mixtures}

Mixture M13 (mass fraction): 0.124 Butylbenzene, 0.341 butylcyclohexane, 0.259 dodecane, 0.276 isocetane

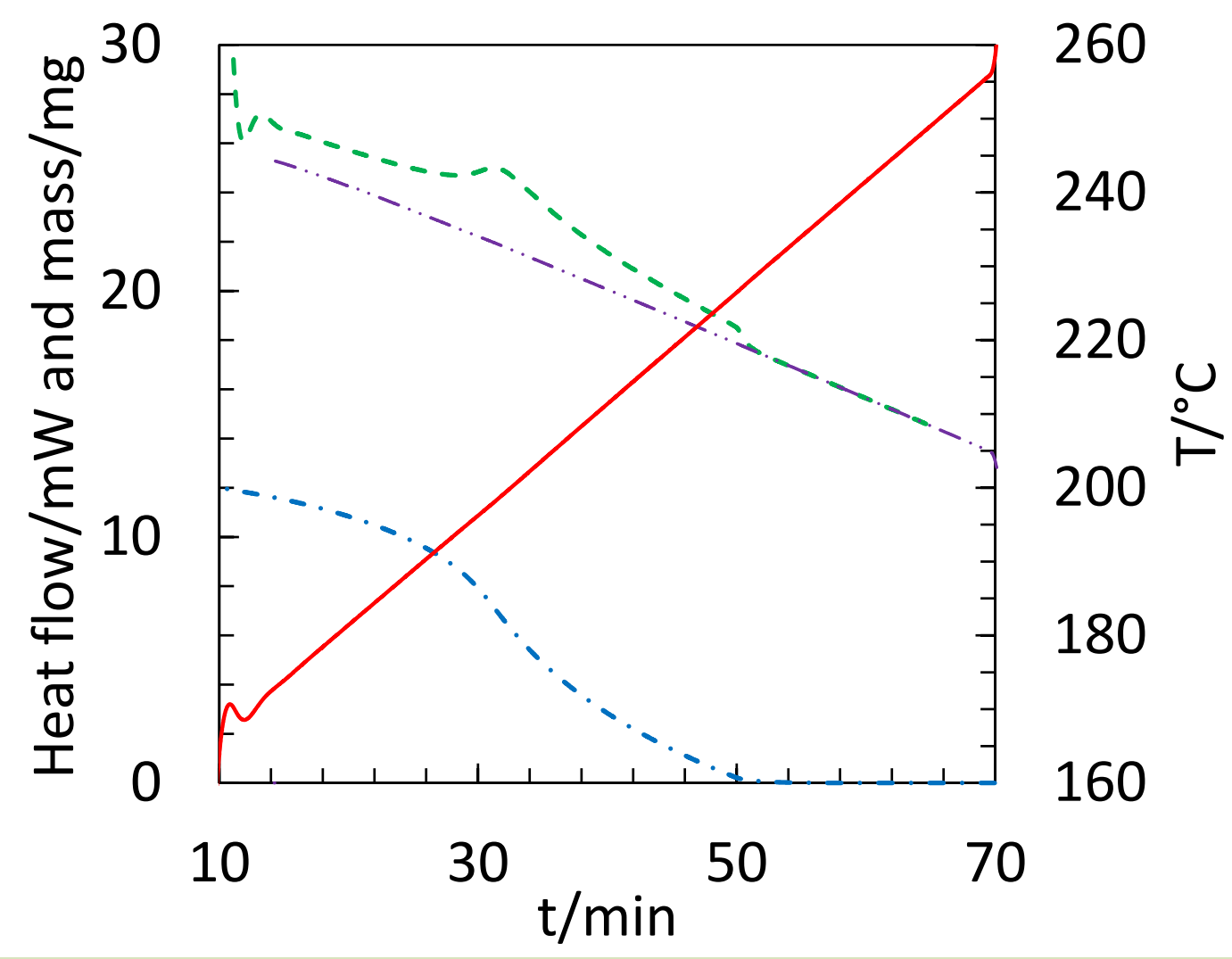

Heat for DSC/TGA heat to raise temp (from heat capacity)

HOV of M13

$238 \pm 7 \mathrm{~J} \cdot \mathrm{g}^{-1}$

matches

JP-5: $239 \pm 8 \mathrm{~J} \cdot \mathrm{g}^{-1}$ 


\section{Temperature ramping: surrogate mixtures}

\begin{tabular}{|c|c|c|}
\hline Mix & $\begin{array}{c}\text { Heat } \\
\text { capacity } \\
\left(\mathrm{J} / \mathrm{g} \cdot{ }^{\circ} \mathrm{C}\right)\end{array}$ & HoV \\
\hline $\mathrm{J} / \mathrm{g})$
\end{tabular}

\begin{tabular}{|c|c|c|}
\hline Mix & $\begin{array}{r}\text { Heat capacity } \\
\left(\mathrm{J} / \mathrm{g}{ }^{\circ} \mathrm{C}\right)\end{array}$ & $\begin{array}{c}\text { HoV } \\
(\mathrm{J} / \mathrm{g})\end{array}$ \\
\hline $\mathrm{JP}-5$ & $\mathbf{1 . 9 1}$ & $239 \pm 8$ \\
\hline M7 & 1.92 & $239 \pm 11$ \\
\hline M8 & 2.05 & $228 \pm 10$ \\
\hline M9 & 1.93 & $229 \pm 17$ \\
\hline M10 & 1.92 & $249 \pm 14$ \\
\hline M11 & 1.91 & $219 \pm 15$ \\
\hline M12 & 1.89 & $224 \pm 15$ \\
\hline M13 & 1.91 & $238 \pm 7$ \\
\hline M14 & 2.03 & $238 \pm 11$ \\
\hline M15 & 2.03 & $226 \pm 7$ \\
\hline
\end{tabular}

Heat capacity: $25^{\circ} \mathrm{C}$

Mix 6: $50 \%$ by mass Isocetane/dodecane 


\section{Summary and Conclusions}

\section{Developed Isothermal method using DSC/TGA}

- HOV of pure compounds matched literature

- No method could be made for jet fuel

Developed Temperature ramping method DSC/TGA

- Utilized using 'Hi Res Dynamic' method on DSC/TGA

- Measured heat capacities using DSC

- HOV of pure compounds similar to values from isothermal

- HOV of JP-5 fell in range of estimates based on correlations

- Three 4-component surrogates $\rightarrow$ low HOV compared to JP-5 (had not combusted well in previous studies)

- Other 4-component surrogates matched JP-5 within $90 \%$ confidence interval 


\section{Questions}

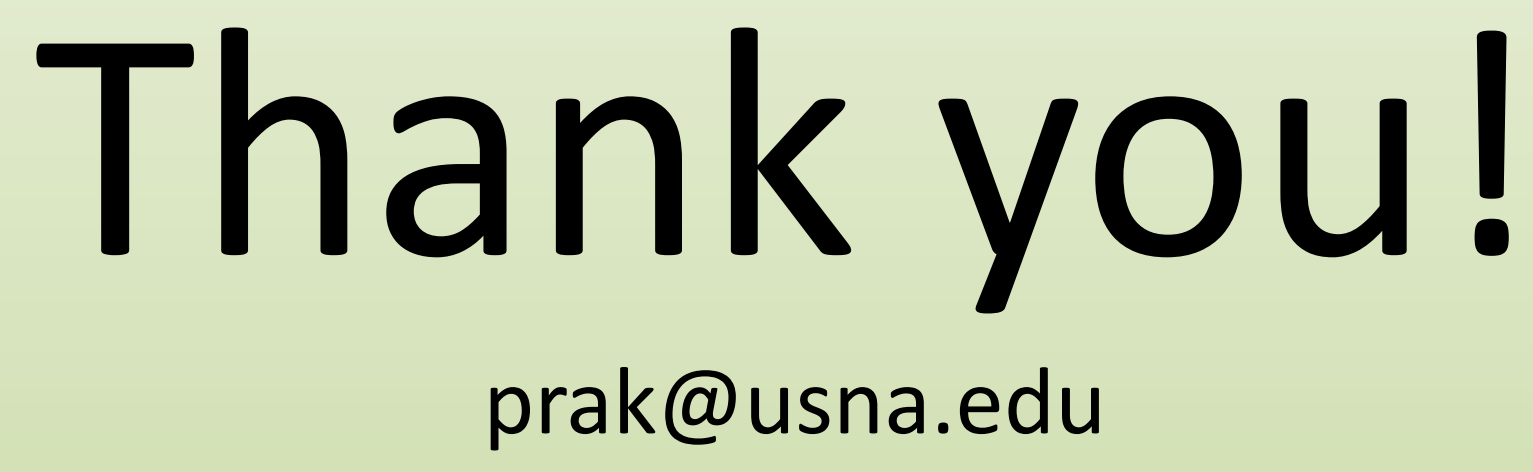

